\title{
Traducción de literatura poscolonial en lengua portuguesa
}

«Le pesaba en el cuerpo Europa entera»

\section{Rebeca Hernández}

\section{OpenEdition}

\section{Journals}

Edición electrónica

URL: https://journals.openedition.org/cher/970

DOI: $10.4000 /$ cher. 970

ISSN: 2803-5992

\section{Editor}

Presses universitaires de Strasbourg

\section{Edición impresa}

Fecha de publicación: 12 diciembre 2019

Paginación: 143-155

ISBN: 979-10-344-0057-7

ISSN: 1968-035X

\section{Referencia electrónica}

Rebeca Hernández, «Traducción de literatura poscolonial en lengua portuguesa», reCHERches [En línea], 23 | 2019, Publicado el 27 septiembre 2021, consultado el 18 noviembre 2021. URL: http:// journals.openedition.org/cher/970 ; DOI: https://doi.org/10.4000/cher.970 


\title{
Traducción de literatura poscolonial en lengua portuguesa "Le pesaba en el cuerpo Europa entera»
}

\author{
ReBECA HeRNÁNDEZ ${ }^{1}$
}

\section{La traducción de la literatura poscolonial en lengua portuguesa}

La práctica de la traducción al español de la literatura poscolonial en lengua portuguesa se desarrolla, por diversos motivos, en lo que podría denominarse un ámbito de la excepción. En primer lugar, por tratarse esta de una literatura de reciente aparición, ya que, a pesar de que sus primeras manifestaciones surgen a finales del siglo XIX, no comienza a desarrollarse de forma plena hasta la segunda mitad del siglo xx. En segundo lugar, por la indudable experimentación, tanto lingüística como relativa a los modelos literarios occidentales heredados por los territorios colonizados, que la distingue y articula con el fin de llevar a cabo una afirmación de la identidad propia y de lucha contra el poder colonial. En tercer lugar, constituye esta literatura una excepción por su presencia periférica dentro de la industria editorial en español. Tomando esto como base, se presentan, a continuación, algunas reflexiones sobre las circunstancias de excepcionalidad que acabamos de referir, desde la aparición de esta literatura hasta nuestros días, así como la pertinencia de que sea vista o no, ella misma, como una excepción. En este sentido, cobra especial relevancia la entrada dedicada a la traducción de las literaturas africanas en lengua portuguesa que puede encontrarse en el Diccionario histórico de la traducción en España de Lafarga y Pegenaute, de la autoría de Pere Comellas, uno de los más destacados traductores de literaturas africanas de lengua portuguesa al castellano y al catalán:

El interés por las literaturas africanas de lengua portuguesa en España ha sido en general minoritario. Se trata de literaturas sujetas a una doble marginación, por el hecho de surgir en un espacio cultural considerablemente ignorado, África, y

1 Rebeca Hernández, Universidad de Salamanca, ORCID: 0000-0003-0671-8577 
en una lengua que en los cánones literarios europeos tradicionales tiene posición periférica. (2009: 929)

«Le pesaba en el cuerpo Europa entera», la frase que da título a este texto, pertenece a la novela O Outro Pé da Sereia, de Mia Couto (2006: 243), uno de los mayores escritores de la literatura mozambiqueña. Esta frase ilustra de un modo agudo y gráfico las consecuencias del proceso de colonización llevado a cabo por la civilización europea, que creó una nueva realidad mundial, en su afán de expansión desde el siglo xv hasta el xx por América, África y Asia, con el fin de reproducir su forma de vivir y de entender la realidad en la no civilización. Del mismo modo que el continente europeo marcó la historia colonial de medio mundo, hoy, más de cuatro décadas después de la consecución de las independencias por parte de las antiguas colonias portuguesas en África, se hace necesario reflexionar hasta qué punto Europa y la impronta de la civilización occidental siguen pesando en los procesos de traducción y de recepción de las literaturas producidas en torno a un mismo hecho histórico: la liberación de los actuales países africanos de lengua oficial portuguesa y la guerra colonial de Portugal en África.

En 1415, con la toma de Ceuta por parte de Portugal, comienza la presencia de los portugueses en África. Tras siglos de exploración, de ocupación y de explotación del terreno y de su población en diferentes puntos geográficos, sobre todo costeros, se inicia, tras la Conferencia de Berlín que tuvo lugar entre los años 1884 y 1885, una carrera contrarreloj por intentar controlar parte del interior del continente africano y abrir un corredor entre los actuales Angola y Mozambique. Como es bien sabido, la Conferencia de Berlín supuso el punto de partida para el reparto de África entre las naciones europeas colonizadoras y, en los años siguientes a su celebración, se constituyeron los territorios que serían, primero, colonias portuguesas y, más adelante, provincias ultramarinas durante el Estado Novo, periodo que se desarrolló entre 1933 y 1974 bajo la dictadura de Salazar (1933-1968) y Caetano (1968-1974). En 1975, después de una cruenta guerra colonial que tuvo lugar entre 1961 y 1974 y que culminó el 25 de abril de ese último año con la Revolución de los Claveles, estos territorios se convirtieron en las hoy naciones independientes de Angola, Mozambique, Guinea Bissau, Cabo Verde y Santo Tomé y Príncipe. La producción literaria que refleja la denuncia al sistema colonizador y el deseo, la intención y la lucha por conseguir la independencia de la opresión colonial recibe la denominación de literatura poscolonial y engloba no solo a la creación de aquellos autores pertenecientes a los territorios africanos sino también a aquellas voces provenientes de la nación colonizadora que muestran, en sus textos, un profundo rechazo hacia la política imperial de su país. En este artículo presentaremos una serie de cuestiones relacionadas con esta producción literaria poscolonial y su traducción al español. Todo esto comporta que, dentro de la actividad de traducción de las literaturas africanas, el terreno por el que se transita es, como ya hemos apuntado, el de la excepción. 


\section{Literatura y excepción}

Antes de hacer referencia al caso concreto de las obras literarias poscoloniales en lengua portuguesa, es necesario apuntar que la literatura, como forma de arte particular, constituye, en sí misma, una región propicia para la excepción. Para quien escribe, la literatura conlleva la creación de un espacio y de un estilo propio, la construcción de un proyecto estético, en el que reflejar, a través de la lengua como materia dúctil, la relación particular con la memoria, la realidad o la invención. Para quien lee, la relación que entabla con la literatura supone establecer una red que aúne la experiencia estética, los símbolos, las metáforas con la estructura intelectual propia, la que existe previamente y con lo que está por construir. En los cimientos de esta construcción de lo excepcional tanto en la labor creativa de los escritores como en el proceso de recepción de los lectores han de estar la novedad y la naturaleza de lo extraordinario.

Las particularidades de la lengua literaria y del estilo literario han sido ampliamente pensadas, argumentadas y definidas a lo largo de la Historia de la Teoría Literaria, así como también lo han sido los procesos de recepción de los lectores; del mismo modo, la relación del traductor con la literatura que traduce, la comunicación que se genera con el texto de origen y la creación del texto meta han sido, y son, objeto de reflexión, de ensayos, de debate en el ámbito de los estudios de traducción.

En este sentido, y en lo que a la traducción literaria se refiere, podemos tomar como un punto de inicio el hecho de que traducir literatura es un acto de creación controlado. La voz del traductor crea, pero lo hace dentro de unas condiciones, que son las que ha establecido el autor en su obra original. Resulta posible afirmar que la traducción literaria es un juego en el que existe una serie de reglas marcadas por el escritor que el traductor ha de seguir a través de la duda, de la reflexión y de la toma de decisiones; para todo ello, hay que tener en consideración elementos tales como el contexto de la escritura de la obra, el lugar que ocupa en una determinada literatura, el periodo histórico en el que se inscribe o el proyecto literario y estético del escritor. Del mismo modo, y dentro de una escala más puramente microtextual, la atención ha de recaer sobre los elementos gramaticales, léxicos o prosódicos que construyen la obra que se está traduciendo.

Ahora bien, una vez aceptadas las reglas del juego del escritor con la creación literaria y del traductor con la creación literaria controlada, queremos ofrecer una reflexión acerca de la traducción de la ya citada literatura poscolonial en lengua portuguesa. Como se ha avanzado en líneas anteriores, esta etiqueta concierne a la literatura creada en los cinco países africanos de lengua oficial portuguesa: Angola, Mozambique, Cabo Verde, Guinea Bissau y Santo Tomé y Príncipe, colonias de Portugal hasta 1975; y a la literatura portuguesa que reflexiona sobre el fenómeno colonial y lo denuncia.

En el caso de las naciones africanas, cada uno de estos países, con sus especificidades geográficas, etnográficas, sociales y lingüísticas, ha buscado 
crear desde finales del siglo XIX su propia literatura, representante de una identidad específica y alejada tanto de la literatura producida en Portugal como de los modelos literarios europeos. Así, las literaturas de estos cinco países han servido para censurar la presencia colonial de los portugueses en África y han acompañado los diferentes procesos de liberación de estos territorios africanos. Del mismo modo, los autores portugueses que cuestionan en sus obras la historia de su país como potencia imperial plantean en sus creaciones la necesidad de revisar la relación de Portugal con África desde una perspectiva de empatía, de crítica y de denuncia. No hay que olvidar que, dentro del proceso de descolonización de las naciones africanas, se puede observar aquello que el sociólogo portugués Boaventura de Sousa Santos definió como «un sentido compartido de liberación, tanto para el colonizador como para el colonizado» (2001:73). De hecho, como es bien sabido, el régimen de la dictadura militar del Estado Novo portugués llegó a su fin ante la insostenible situación de Portugal en sus colonias africanas, y gracias a la acción de las propias fuerzas militares portuguesas en la Revolución de los Claveles, el 25 de abril de 1974.

En este sentido, resulta pertinente preguntarse qué sucede cuando una obra literaria surge en una situación que puede considerarse de excepción a su propio proceso de creación, cuando a la acción de escribir se le suma una denuncia, una reivindicación, una lucha de la que forman parte no solo el escritor sino toda su comunidad. Igualmente clave sería preguntarse cómo afronta el traductor una obra literaria escrita en un momento convulso de la historia, en el que la vida está en juego, con la violencia instalada en la cotidianeidad, la censura sobrevolando la expresión de cada idea. En estas circunstancias, el hecho literario se ve condicionado por la necesidad de creación de un nuevo modo de entender la realidad, de representarla, de escribir entre líneas como parte de un proyecto de construcción de una nueva identidad capaz de superar la presencia continuada de la colonización.

\section{Poscolonialismo, literatura y traducción literaria}

En el ámbito de las Humanidades y de las Ciencias Sociales, el fin de la presencia colonial de las potencias europeas en África y Asia ha venido acompañado por el surgimiento de los denominados estudios poscoloniales en las instituciones académicas de Occidente. A través de esta corriente de pensamiento, se han fomentado el análisis y la reflexión sobre los diferentes modos de colonización cultural e intelectual por parte de las naciones de Europa en los territorios colonizados y, a su vez, se han estudiado y categorizado las estrategias de resistencia lingüística e identitaria a los procesos de colonización europeos por parte de estos territorios. Así, y a través de esta rama de estudios, se ha procedido a la observación y a la descripción de los diferentes mecanismos de apropiación y subversión, tanto de la lengua como de la cultura impuestas por Occidente, llevados a cabo por los territorios colonizados como forma de confrontar los intentos de civilizar y de abolir identidades por parte de 
la colonización europea. Dentro del poscolonialismo, han surgido trabajos fundamentales de intelectuales como Bhabha (1994), Spivak (1999), Said (1993) o Ashcroft, Griffiths y Tiffin (1989, 2000), que han servido para trazar, por una parte, los mecanismos culturales e identitarios de colonización de las naciones europeas y, por otra, las herramientas de resistencia empleadas por los territorios colonizados. Otros estudios imprescindibles para entender el orden mundial derivado de los procesos de colonización han sido los realizados por Aníbal Quijano (2000) y Walter Mignolo (2003, 2005) y sus lecturas decoloniales del orden mundial, o Boaventura de Sousa Santos, quienes, fuera del ámbito anglófono, han trazado una anatomía clarividente de las características y las consecuencias de los colonialismos de raíz ibérica dentro del contexto global. De la mano de estas reflexiones sobre el poscolonialismo y la descolonización, ha surgido en las instituciones académicas occidentales, desde las últimas décadas del siglo $\mathrm{xx}$, un sentido crítico de los procesos de colonización por parte de las naciones europeas, así como una conciencia de la perspectiva eurocéntrica que ha dominado, en la mayor parte de los casos, la producción de pensamiento y de cultura en Occidente. A la luz del poscolonialismo, las herramientas occidentales se han diluido para producir una lectura crítica de las consecuencias que el colonialismo ha tenido en la historia, en la sociedad, en las humanidades, en la literatura y en el arte de los países colonizados.

Por otra parte, los estudios poscoloniales y los de traducción literaria han tenido también una relación fecunda en las últimas décadas. Así, la perspectiva traductológica ha permitido entender la literatura poscolonial como un ejercicio de traducción literaria, ya que sus autores, en la mayor parte de ocasiones hablantes nativos de lenguas africanas, han optado por escribir sus obras en las lenguas europeas impuestas en sus respectivos territorios colonizados. Esta elección lingüística presenta, como una de sus consecuencias más directas, la posibilidad de que las ideas de denuncia y de construcción identitaria contempladas en sus libros obtuviesen una mayor difusión internacional. Este fin se alcanzaría a través del ejercicio de traducción entre lenguas, culturas y modelos culturales llevado a cabo por los propios autores.

Además, y en paralelo, los estudios de traducción han construido su propia rama ligada al poscolonialismo, que ha contribuido a la formación de una estructura ética por parte de los traductores occidentales de estas obras a una tercera lengua. A través del conocimiento de cuestiones relativas a la historia, a la sociedad y a las identidades surge una conciencia ética que nace del conocimiento de todas las especificidades de estas literaturas en el momento de importarlas a un nuevo sistema literario occidental, dentro de las reglas de poder y jerarquía que rigen la que Pascale Casanova denominó República mundial de las Letras:

La descolonización impulsó a todos los países excluidos hasta entonces de la noción misma de literatura propia (en África, en la India, en Asia...) a reivindicar a su vez el acceso a la legitimidad y a la existencia literaria. (2001:24) 
El acto de traducir y editar estas literaturas en Occidente supone una acción delicada, en la que el riesgo de realizar una traducción exotizante adquiere una nueva dimensión que obliga a desplegar, más que nunca, un juego de equilibrio que dé buena cuenta al lector del texto meta de todos los factores políticos, sociales y jerárquicos que influyeron en el proceso de creación, y que no convierta la traducción en un texto exótico, fruto de una realidad exótica. Esta tensión por incorporar el texto de origen en la cultura de recepción, con todo su contenido de denuncia y de afirmación de la identidad, se hace visible, en muchas ocasiones, a través de la inclusión de paratextos en los libros traducidos.

\section{La excepción dentro de la literatura poscolonial de lengua portuguesa y la excepción en su traducción al español. Estudio de dos casos}

La literatura producida en torno a los procesos de liberación de las antiguas colonias portuguesas, en su contexto previo, en el durante, o en el momento posterior a estas luchas, supuso una modificación en los ámbitos literarios africano y portugués. Si hasta 1975, solo existía la literatura portuguesa a la que, como mucho, se le podía añadir la etiqueta de ultramarina, a partir de esta fecha van a surgir nuevos sistemas literarios independientes para cada una de las nuevas naciones: Angola, Mozambique, Guinea Bissau, Cabo Verde y Santo Tomé y Príncipe. Estas nuevas literaturas no solo englobarán las obras producidas a partir de 1975, sino que, de forma retrospectiva, incluirán también todas aquellas creaciones fruto de la confrontación con el poder colonial. En este mismo sentido, además, y al tiempo que comienza a coexistir con los nuevos sistemas literarios que se encuentran en plena fase de aparición, la literatura portuguesa creará una serie de obras en torno a la guerra colonial, reflejo de esta situación histórica de escisión y trauma.

Toda esta creación literaria, la africana y la portuguesa de la guerra colonial, encontrará su proyección en el exterior, ya que, de forma ineludible, comenzará a ser traducida y exportada dentro de los circuitos de mercado de la industria editorial.

Es habitual que las obras literarias producidas tanto en las antiguas colonias portuguesas en África como en Portugal, antes, durante o después de los procesos de liberación, comporten una serie de características discursivas relativas a la realidad identitaria de los territorios colonizados. Así, y dentro de la producción literaria perteneciente a este contexto histórico, que, como ya hemos dicho, acompaña la lucha por la independencia y empatiza con ella, vamos a poner la atención sobre dos obras literarias del siglo $\mathrm{xx}$ escritas en lengua portuguesa, que servirán para ilustrar estas especificidades y la manera cómo fue abordada su traducción al español por quien firma este artículo. Ambos libros pertenecen a la denominada literatura poscolonial, por cuanto reflexionan sobre las situaciones derivadas del poder imperial portugués y las denuncian. La primera de estas obras es el conjunto de relatos Nós matámos o Cão-Tinhoso, del 
escritor mozambiqueño Luís Bernardo Honwana, la segunda, la novela Autópsia de um mar de ruínas, del escritor portugués João de Melo. El contexto histórico de estas dos obras es el mismo: la lucha por la independencia de las antiguas colonias de Portugal en África, que tuvo lugar entre los años de 1961 y 1974.

De este modo, las dos obras referidas de Luís Bernardo Honwana y João de Melo denuncian la historia colonial y política de Portugal en el continente africano, así como las injusticias sociales derivadas de esta situación. Puede afirmarse que ambas obras, por su naturaleza literaria y discursiva, como a continuación veremos, constituyen una excepción dentro de sus respectivos sistemas literarios.

\section{Nós Matámos o Cão-Tinhoso, de Luís Bernardo Howana y el portugués normativo}

Nós Matámos o Cão-Tinhoso, del escritor mozambiqueño Luís Bernardo Honwana, es, como ya se ha apuntado, una obra de relatos. Está compuesta por siete cuentos y las circunstancias de su escritura y de su edición fueron excepcionales debido a diversos motivos. La obra fue publicada en 1964, año en el que se iniciaron las luchas de liberación nacional en la entonces colonia portuguesa de Mozambique. Ese mismo año, Honwana fue hecho prisionero por la PIDE (Policía Internacional y de Defensa del Estado de Portugal). Por otra parte, Nós Matámos o Cão-Tinhoso es la primera obra narrativa publicada en Mozambique escrita por un mozambiqueño, por lo que supuso no solo el debut literario de su autor, a los 22 años, sino también la inauguración de la narrativa de este país africano cuando era aún colonia portuguesa. Además, los relatos se tradujeron al inglés ${ }^{2}$ y fueron publicados en 1969 en la emblemática colección Heinneman's African Writers Series, lo que permitió que la situación que se vivía en el Mozambique colonial fuese difundida en el exterior. Se trata de un libro en el que hay un trabajo estilístico visible, que, significativamente y según la tradición crítica imperante, se ha descrito a través de herramientas occidentales. De la obra de Honwana se ha referido que sus cuentos siguen la senda de escuelas literarias europeas como el neorrealismo o el nouveau roman (Laranjeira 1995: 291). Los siete cuentos que componen la obra describen de una forma sutil y, en muchas ocasiones, a través de los silencios y de aquello que no se dice, la realidad del Mozambique colonial. En su obra, nos muestra Luís Bernardo Honwana la vida de los niños, de los jóvenes, de las mujeres, de los ancianos..., en un contexto de opresión al que no resulta fácil hacer frente y en el que se ha de buscar cómo acallar la rabia y el dolor que esta situación comporta. Al mismo tiempo, el autor mozambiqueño dibuja a través de sus cuentos una esperanza de futuro asentada en la infancia y en la juventud, y en la posibilidad de encontrar, a través de la afirmación individual contra la opresión, un camino capaz de cambiar la situación colonial. Desde un punto de vista lingüístico, algo que también ha destacado la crítica con relación a la obra de Honwana,

2 La autora de la traducción al inglés del libro de Luís Bernardo Howana, We Killed Mangy Dog, fue Dorothy Guedes. 
autor bilingüe en portugués y ronga, es la elección de una lengua portuguesa normativa para escribir sus relatos, exceptuando la utilización puntual de algunas palabras o de algunas referencias a las lenguas africanas habladas en el territorio mozambiqueño. Este léxico autóctono aparece incluido dentro del discurso en portugués, sin ningún tipo de señalización tipográfica o paratextual que explique el significado de esas palabras y, en varias ocasiones, existen referencias por parte del narrador al hecho de que algunos de los personajes hablan en una lengua que no es el portugués, a pesar de que en los cuentos esas intervenciones en cuestión aparecen traducidas al portugués. Veamos algunos ejemplos de este proceso de traducción de los personajes por parte del autor.

En el primer caso, encontramos la intervención de uno de los personajes, una mujer bilingüe en portugués y en ronga que cambia de lengua según el interlocutor al que se dirige, por una parte, su hijo y, por otra, la mujer que la ayuda en casa con las tareas domésticas. Como se puede apreciar, todo el parlamento, perteneciente al cuento titulado «Papá, cobra e eu», está redactado en portugués y presenta la indicación del narrador del cambio de código lingüístico del personaje:

Tu, Ginho (agora falava em portugués) toma conta da casa e lembra-te de que já não és nenhuma criança [...] Sartina (voltou a falar em ronga) quando acabares isso põe a chaleira ao lume para o lanche das crianças [...] Ginho (agora era em portugués), toma conta de tudo que eu volto já. (2000: 97, mis cursivas)

También incluye Honwana, dentro del discurso en portugués, algunos términos o frases provenientes de lenguas mozambiqueñas. Cada una de las palabras incluidas en el discurso en portugués funciona como una llamada de atención al lector de la obra, al incidir en el hecho de que el portugués fue una lengua impuesta y de que hay otras lenguas, como el ronga, el swazi o el changana, que son las que habla la población colonizada. Funcionan estas palabras en el contexto de escritura de la obra como una sinécdoque de la realidad mozambiqueña. En este sentido, es necesario resaltar que, en la obra original, estas palabras no aparecen marcadas de ninguna manera, así como tampoco existe ningún paratexto que haga referencia a ellas, como sucede en el siguiente ejemplo:

Erguendo as mãos até à altura da cabeça [...] saudou o administrador:

- Bayeti n’kossi!... (2001: 127)

La traducción al español de esta obra, publicada en 2009, mantiene las palabras autóctonas de las lenguas mozambiqueñas dentro del discurso traducido. No obstante, como criterio editorial se optó por marcarlas en letra cursiva e incluir un glosario al final del libro para que, el lector que así lo desease, pudiera consultar su definición. Así, la oración citada fue traducida del siguiente modo:

Levantando las manos hasta la altura de la cabeza [...] saludó al administrador: - Bayeti n'kossi!... (2009: 150)

En el glosario: 
Bayeti n'kossi: Fórmula ceremoniosa de saludo a un rey, aceptación de la autoridad de alguien a quien nos dirigimos o sumisión a una sentencia o decisión que nos afecte. Esta expresión procede de la lengua zulú, pero empezó a utilizarse en el sur de Mozambique tras las invasiones Nguni en los siglos XVII y XVIII. (2009: 165)

Otro ejemplo de la inclusión de la lengua ronga dentro del discurso en portugués puede verse en el fragmento que reproducimos a continuación:

[O] Papá começou:

- Tatana, ha ku dumba Hosi ya tilo ni misaba (2001: 104)

La traducción al español se realizó del siguiente modo:

Papá empezó a rezar el padrenuestro en ronga:

-Tatana, ha ku dumba Hosi ya tilo ni misaba (2009: 126)

Como se puede apreciar, se optó por introducir una glosa intratextual en la traducción, se especifica dentro del texto el significado de la frase y la lengua en la que está escrita. El hecho de mantener en la traducción todas las palabras en las lenguas autóctonas habladas en Mozambique, resaltarlas en cursiva y explicarlas en un glosario al final del libro dejando en manos del lector la decisión de consultar su significado, permite mostrar de un modo efectivo la realidad plurilingüe de esta nación africana. Al mismo tiempo, estas decisiones traductológicas tienen como objetivo resaltar la imposición lingüística, cultural y religiosa por parte de la colonización portuguesa, que puede ser ajena al conocimiento del lector de la obra en castellano.

\section{Autópsia de um Mar de Ruínas, de João de Melo y la africanización del portugués}

En el caso de la segunda obra que vamos a considerar, Autópsia de um Mar de Ruínas, encontramos una novela de João de Melo publicada en 1984 y situada en Angola durante el periodo de la Guerra Colonial. João de Melo, uno de los grandes escritores portugueses contemporáneos, nació en la isla azoriana de São Miguel en 1949, y en 1970 se vio obligado a desplazarse a Angola al ser movilizado como parte de los servicios sanitarios del ejército portugués. Esta novela constituye una disección de la caída del imperio portugués en la guerra colonial y se erige como una de las obras primordiales de este periodo histórico y literario. La novela describe el día a día de la localidad angoleña de Calambata durante la guerra colonial y retrata la vida de los soldados portugueses y de los habitantes angoleños en torno a una sanzala o aldea de africanos controlada por los portugueses. A través de este contexto, João de Melo muestra la barbarie del colonialismo portugués en África y realiza una dura crítica a la política del Estado Novo de Salazar y Caetano. En esta novela, su autor no se limita a ofrecer la perspectiva de este conflicto a través de la voz de los soldados portugueses, sino que también desdobla su voz para mostrar el punto de vista de los colonizados angoleños. João de Melo lleva a cabo este desdoblamiento mediante una serie de recursos estilísticos y lingüísticos. Así, si en los capítulos impares tiene un protagonismo la perspectiva de los soldados portugueses, en 
los capítulos pares la voz preponderante es la de los colonizados africanos. João de Melo consigue dotar de especificidad a ambas voces a través del uso de la lengua, ya que la gramática de aquellos personajes angoleños que se expresan en portugués aparece fuertemente influenciada por las lenguas africanas.

Si Nós Matámos o Cão-Tinhoso se relacionaba en su forma con ciertas corrientes estéticas occidentales y presentaba un portugués marcado por la norma europea de esta lengua, es posible entroncar esta novela de Melo en la tradición de la literatura angoleña. El autor portugués sigue en ella los pasos literarios de otros escritores angoleños como Luandino Vieira y Boaventura Cardoso, y utiliza numerosos recursos discursivos para africanizar el portugués de sus personajes y destacar, de este modo, que la lengua europea fue un idioma impuesto sobre las lenguas africanas.

Uno de los recursos utilizados por João de Melo para este fin es la introducción de términos léxicos procedentes de las lenguas habladas en Angola, que se da de un modo constante en esta novela, como vemos a continuación:

Tinha, porém, a esperança das suas crianças, comia a fuba e a moamba que as mulheres lhe davam a provar em testos de barro (2002: 55-56)

Es posible constatar cómo en el original portugués, João de Melo decide, al igual que Luís Bernardo Honwana en Nós Matámos o Cão-Tinhoso, no resaltar de ninguna manera ni definir las palabras originarias de las lenguas de Angola, quimbundo y kikongo. Sin embargo, en la traducción al español, con el criterio editorial de acercar la realidad descrita en la obra al lector ajeno, en la mayor parte de los casos, a la presencia portuguesa en África y a la realidad de los procesos de liberación, sí se decidió marcarlas en cursiva y proporcionar su traducción en el glosario incluido al final de la edición, como se puede apreciar a continuación:

Tenía, sin embargo, la esperanza de sus niños, comía la fuba y la moamba que las mujeres le daban a probar en vasijas de barro (2017: 57)

En el glosario:

Fuba: harina de mandioca o maíz con la que se hacen gachas (2017: 306)

Moamba: guiso de gallina realizado con ingredientes como pimienta de tabasco y fungi (2017: 306)

Es también frecuente que João de Melo africanice la lengua portuguesa recurriendo a un uso agramatical de la misma, marcada por la influencia de las lenguas quimbundo y kikongo. En estos casos, se reprodujeron algunas de las estrategias discursivas del texto en portugués en la traducción al español, aunque no con tanta presencia como en el texto original. Esto fue así porque, si bien el original portugués reproduce la lengua portuguesa fuertemente influenciada por la sintaxis y el léxico de las lenguas angoleñas autóctonas, el trasladar esta agramaticalidad al español con la misma presencia que tiene en el texto original, sería crear una nueva lengua, sin un referente lingüístico existente que podría llegar a alejar al lector de la obra de la realidad descrita en la novela. No obstante, 
sí se optó por reproducir una cierta agramaticalidad. Así, en el siguiente ejemplo, vemos cómo el autor emplea la preposición em, contraída con el artículo indeterminado masculino singular $u m$, en un contexto lingüístico no normativo, en sustitución de la preposición com, que sería la adecuada para este caso:

Nunca mais vai ter amizade assim num branco, papá. (2016: 59, mis cursivas)

En este caso, el uso agramatical de la preposición se reproduce en la traducción al español:

Nunca más tiene amistad así en un blanco, papá (2017: 57, mis cursivas)

También encontramos otros ejemplos que constituyen una excepción en el discurso normativo en portugués, como la siguiente situación en la que el comparativo de superioridad pior viene antecedido por el cuantificador mais.

Para ele, a mentira era ainda mais pior que uma traição (2002: 359)

En esta oración, la estructura agramatical se tradujo al castellano como más peor:

Para él, la mentira era aún más peor que una traición (2017: 297)

Además de la incorporación de algunas de las estrategias discursivas empleadas en el original, la edición en español también contó con una serie de paratextos que sirven para situar al lector en la situación retratada en la novela. Así, presenta un prólogo en el que se desarrollan las circunstancias de la guerra colonial de los portugueses en África y se contextualiza la obra dentro de la realidad que describe y su relación con el proyecto literario de otros escritores angoleños. Además del glosario ya citado, recoge una nota a la traducción en la que se explican las características léxicas y sintácticas del texto original como modo de subrayar la realidad colonial en Angola y, también, incluye una cronología biográfica de João de Melo y una bibliografía referente al autor portugués y a la literatura de la guerra colonial. De este modo, el lector puede tener acceso a la compleja realidad de la que es fruto esta novela y a la perspectiva del autor, contrario al régimen dictatorial que marcó la vida en Portugal durante cinco décadas.

\section{Conclusión: ¿Cuándo deja la excepción de constituir una excepción?}

A lo largo de este texto, hemos observado que la traducción de las obras literarias poscoloniales en lengua portuguesa se desarrolla bajo el signo de la excepción y de lo excepcional: los autores poscoloniales buscan diferentes modos de denuncia que no han de ser obviados en sus traducciones y ediciones en otras lenguas, bien a través de los mismos recursos lingüísticos utilizados por ellos, bien a través de la inclusión de elementos paratextuales.

Ahora bien, una vez que han pasado cuatro décadas desde la obtención de las independencias por parte de los países se hace necesario reflexionar acerca de dónde se sitúa ahora, en la literatura creada en la actualidad, el grado de búsqueda o de reafirmación de la identidad propia, o si las literaturas producidas en los cinco países africanos de lengua oficial portuguesa han entrado en la que 
podríamos definir como una fase de normalización. Es decir, si en las obras escritas en torno a los procesos de independencia, consideramos que cada palabra que aparece en una lengua africana dentro del discurso en portugués funciona como una sinécdoque de una identidad oprimida, en la actualidad, ¿esto sigue siendo así o por el contrario se trata de un término que forma parte de la lengua portuguesa del día a día en ese determinado territorio? Son estas consideraciones las que deberán meditarse al realizar una traducción. ¿Cuándo han de mantenerse las palabras utilizadas por el autor en las diferentes lenguas africanas? Se hace necesario pensar si el uso africanizado de la sintaxis, o la inclusión de una determinada palabra africana, se trata de una reivindicación o de una llamada de atención que muestra que el portugués es una lengua impuesta o si la conservación de los términos africanos o de la agramaticalidad en la traducción va a servir para exotizar al autor y a su obra, para señalar lo pintoresco, lo exótico que ese texto tiene para el lector occidental. ¿Podemos afirmar que, en la literatura escrita hoy, estamos ante el portugués de Angola, de Mozambique, de Cabo Verde, de Guinea Bissau o de Santo Tomé y Príncipe y traducirlo como un todo a una tercera lengua? La principal cuestión es conocer cuáles son los indicadores de que el uso de las lenguas africanas en una obra literaria ha de ser visto como algo excepcional, que exige tomar medidas de excepción en la traducción tales como marcas gráficas o paratextos, o cuándo deja lo excepcional de constituir una excepción y pasar a ser tratado como algo normal, en el sentido de que pertenece a una norma lingüística globalmente aceptada. En el contexto internacional de la industria editorial, en el que las traducciones y la circulación de obras entre sistemas literarios son una constante, estas cuestiones deberán ser consideradas objeto de debate.

\section{Bibliografía}

Ashcroft B., Griffiths G. y Tiffin H., 1989, The Empire writes back: theory and practice in post-colonial literatures, Londres, Routledge.

Ashcroft B.etal., 2000, Post-Colonial Studies: The Key Concepts, Londres, Routledge. Bhabha H. K., 1994, The Location of Culture, Londres, Routledge.

Casanova P., [1999] 2001, La República mundial de las Letras, Barcelona, Anagrama, «Argumentos», trad. J. Zulaika.

Couto M., 2006, O Outro Pé da Sereia, Lisboa, Caminho.

Comellas P., 2009, «Portuguesa de África, Literatura» in Lafarga F. y Pegenaute L.,

Diccionario histórico de la traducción en España, Madrid, Gredos, p. 929-932. Honwana L. B., [1964] 2000, Nós matámos o Cão Tinhoso, Porto, Afrontamento. Honwana L.B., [1969] 1987, We Killed Mangy-Dog and Other Mozambican Stories, Harare, Zimbabwe Publishing House, trad. D. Guedes.

Honwana L. B., 2009, Nosotros matamos al Perro-Tiñoso, Madrid, Baobab, trad. R. Hernández. 
Laranjeira P., 1995, Literaturas africanas de expressão portuguesa, Lisboa, Universidade Aberta.

Melo J. de, [1984] 2002, Autópsia de um mar de ruínas, Lisboa, D. Quixote.

Melo J. de, 2017, Autopsia de un mar de ruinas, Bogotá, Uniandes, "Colección Labirinto», trad. R. Hernández.

Mignolo W., 2003, Historias globales/diseños locales, Madrid, Akal.

Mignolo W., 2005, The Idea of Latin America, Malden, Oxford, Victoria, Blackwell Publishing, «Blackwell Manifestos».

Quijano A., 2000, «Colonialidad del poder, eurocentrismo y América Latina» in Lander, E. (org.), La colonialidad del saber: eurocentrismo y ciencias sociales. Perspectivas latinoamericanas, Buenos Aires, CLACSO, p. 122-141.

Said E. W., 1993, Culture and Imperialism, Nueva York, Vintage Books.

Spivak G. C., 1999, A Critique of Postcolonial Reason. Toward a History of the Vanishing Present. Cambridge, MA, Harvard University Press.

Sousa Santos B., 2001, «Entre Prospero e Caliban: Colonialismo, pós-colonialismo e inter-identidade» in Ramalho M.I. y Sousa Ribeiro A. (orgs.) Entre ser e estar. Raízes, percursos e discursos da identidade, Porto, Afrontamento, p. 23-85. 